\title{
Risk of HIV acquisition among circumcised and uncircumcised young men with penile human papillomavirus infection
}

\author{
Anne F. Rositch ${ }^{\mathrm{a}, \mathrm{b}}$, Lu Maoc ${ }^{c}$, Michael G. Hudgens ${ }^{c}$, Stephen Moses ${ }^{\mathrm{d}}$, Kawango Agot ${ }^{\mathrm{e}}$, \\ Danielle M. Backes ${ }^{f}$, Edith Nyagaya ${ }^{g}$, Peter J.F. Snijders ${ }^{h}$, Chris J.L.M. Meijer ${ }^{h}$, Robert C. \\ Bailey', and Jennifer S. Smith ${ }^{\mathrm{j}}$
}

aDepartment of Epidemiology, John Hopkins Bloomberg School of Public Health bepartment of Epidemiology and Public Health, University of Maryland School of Medicine, Baltimore, Maryland 'Department of Biostatistics, University of North Carolina, Chapel Hill, North Carolina, USA ${ }^{\mathrm{d} D e p a r t m e n t s}$ of Community Health Sciences, Medicine and Medical Microbiology, University of Manitoba, Winnipeg, Canada eImpact Research and Development Organization, Kisumu, Kenya fDepartment of Epidemiology, Brown University, Providence, Rhode Island, USA 9Universities of Nairobi, Illinois and Manitoba (UNIM), Kisumu, Kenya hDepartment of Pathology, VU University Medical Center, Amsterdam, the Netherlands 'Division of Epidemiology, University of Illinois at Chicago, Illinois 'Department of Epidemiology, University of North Carolina, Chapel Hill, North Carolina, USA

\section{Abstract}

Objectives-There are very few data from men on the risk of HIV acquisition associated with penile human papillomavirus (HPV) infection and no data on the potential modifying effect of male circumcision. Therefore, this study evaluated whether HPV is independently associated with risk of HIV.

Design-A cohort study of HPV natural history nested within a randomized control trial of male circumcision to reduce HIV incidence in Kisumu, Kenya.

Methods-Prospective data from 2519 men were analyzed using 6-month discrete-time Cox models to determine if HIV acquisition was higher among circumcised or uncircumcised men with HPV compared to HPV-uninfected men.

Results-Risk of HIV acquisition was nonsignificantly increased among men with any HPV [adjusted hazard ratio (aHR) 1.72; 95\% confidence interval (CI) 0.94-3.15] and high-risk HPV (aHR 1.92; 95\% CI 0.96-3.87) compared to HPV-uninfected men, and estimates did not differ by circumcision status. Risk of HIV increased $27 \%$ with each additional HPV genotype infection

(C) 2014 Wolters Kluwer Health | Lippincott Williams \& Wilkins

Correspondence to Anne F. Rositch, PhD, MSPH, Department of Epidemiology and Public Health, University of Maryland School of Medicine, 10 S. Pine St. MSTF 334C, Baltimore, MD 21201, USA. Tel: +1 410706 0071; fax: +1 4107060098 ;

arositch@epi.umaryland.edu.

Conflicts of interest

A.F.R., M.G.H., L.M., S.M., K.A., D.M.B., E.N., and R.C.B. do not have a conflict of interest with this manuscript. C.J.L.M.M. has received speaker fees from GSK and P.J.F.S. has been an advisory board member of GSK. J.S.S. has received consultancy and/or research grants from GSK, Hologic-Genprobe, Qiagen, BD, and Merck Corporation over the past 5 years. 
(aHR 1.27; 95\% CI 1.09-1.48). Men with persistent (aHR 3.27; 95\% CI 1.59-6.72) or recently cleared (aHR 3.05; 95\% CI 1.34-6.97) HPV had a higher risk of HIV acquisition than HPVuninfected men.

Conclusions-Consistent with the findings in women, HPV infection, clearance, and persistence were associated with an increased risk of HIV acquisition in men. Given the high prevalence of HPV in populations at risk of HIV, consideration of HPV in future HIV-prevention studies and investigation into mechanisms through which HPV might facilitate HIV acquisition are needed.

\section{Keywords}

cervical cancer; co-infection; HIV; human papillomavirus; Kenya; male circumcision; vaccination

\section{Introduction}

Human papillomavirus (HPV) is one of the most prevalent sexually transmitted infections (STIs) worldwide, and the cause of anogenital cancers and warts in both men and women $[1,2]$. Recent data among HIV-negative women have shown that cervical HPV infection increases the risk of HIV acquisition [3-6]. In fact, prospective studies have shown that women who recently cleared an HPV infection had the highest risk of HIV acquisition compared to those with no HPV or noncleared HPV infections $[3,6,7]$.

In a previous analysis, limited to examination of only prevalent HPV at study baseline in uncircumcised men, we found HPV was associated with an increased risk of HIV infection over the course of 2 years [8], consistent with the findings from a very limited number of longitudinal studies in men [7,9]. However, given the known impact of male circumcision on acquisition and clearance of HPVand rate of HIV [10,11], it is important to examine the risk of HIV acquisition associated with recent penile HPV infection and clearance in both circumcised and uncircumcised men. Thus, here we build on our previous findings from the randomized control trial (RCT) of male circumcision in Kisumu, Kenya, to determine if young men with current, persistent, or recently cleared HPV infections are at a higher risk for HIV acquisition as compared to men without HPV infection, and to examine the potential modifying effect of circumcision.

\section{Methods}

\section{Study population and design}

From 2002 to 2007, an RCT was conducted in Kisumu, Kenya, to determine the effectiveness of circumcision in reducing the incidence of HIV infection [10]. A cohort study of the natural history of HPV infections in men was nested within this RCT $[12,13]$. Briefly, eligible men were 18-24 years old, uncircumcised, HIV-seronegative, sexually active, and had no current genitourinary symptoms. Of the 2784 men who participated in the RCT, 2533 consented to and were enrolled in the nested HPV study, of which 2519 had HPV DNA and HIV serology results at least once during the study period and were included in the present analysis (1254 immediate circumcision and 1265 delayed circumcision). 
Study visits were conducted at baseline and every 6 months for 24 months for HPV DNA and for 30 months for HIV serology. At each visit, trained male interviewers administered a standardized questionnaire on socio-demographic characteristics and sexual behavior. A physician or clinical officer conducted a physical exam during which a swab (if symptomatic) or urine sample was collected for PCR detection of Chlamydia trachomatis and Neisseria gonorrhea (Roche Diagnostics, Alameda, California, USA). Blood was collected for herpes simplex virus type 2 [HSV-2; ELISA (Kalon Biological Ltd, Aldershot, UK)] and HIV serology. For HPV DNA detection, penile exfoliated cells were collected using prewetted Dacron swabs from two separate anatomical sites: glans, coronal sulcus, and inner foreskin; and shaft and external foreskin [13]. All participants provided informed consent and all study protocols were approved by Institutional Review Boards at each collaborating institution.

\section{Determination of HIV and human papillomavirus infection}

HIV serostatus at baseline through 30 months of follow-up was determined by on-site rapid HIV testing [Determine HIV 1/2 (Abbott Diagnostics, Abbott Diagnostic Division, Hoofddorp, The Netherlands) and Unigold Recombigen HIV (Trinity Biotech, Wicklow, Ireland)], and confirmed with double ELISA [Detect HIV 1/2 (Aldatis, Rome, Italy) and Recombigen HIV 1/2 (Trinity Biotech)]. Blood specimens from all participants who tested positive by at least one rapid test and one ELISA were sent to the Health Canada National HIV Reference Laboratory (Ottawa, Canada) for confirmatory testing by line immunoassay [Inno-Lia HIV 1/2 (Immunogenetics NV, Ghent, Belgium)], as previously described [10]. The HIV seroconversion visit (defined as ' $t$ ') was the first visit at which the participant was confirmed to be HIV-positive.

Human papillomavirus detection was performed on the shaft and glans samples separately, and results were pooled from both anatomical sites for statistical analyses. DNA was isolated from penile cell samples and the presence of human DNA was evaluated by betaglobin-specific PCR. HPV DNA was assessed by GP5+/6+ PCR followed by reverse line blot hybridization to detect $44 \mathrm{HPV}$ types, as described previously [14,15]. Any-HPV refers to infection with any of the 44 genotypes or untyped infections. High-risk HPV (HR-HPV) refers to HPV types $16,18,31,33,35,39,45,51,52,56,58,59,66$, or 68 , regardless of coinfection with low-risk or untyped HPV.

\section{Statistical analyses}

Crude HIV incidence rates were estimated by the number of seroconversions divided by person-months of follow-up. An extended Kaplan-Meier method that accommodates timevarying covariates was employed to nonparametrically estimate and graphically display cumulative risk of HIV seroconversion by HPV infection status at $t-1$ (the visit immediately preceding the HIV seroconversion visit) [16]. Discrete time Cox models were used to estimate adjusted hazard ratios (aHRs) and 95\% confidence intervals (CIs) of the associations between HPV infection at $t-1$ and risk of subsequent HIV acquisition [17]. Any-HPV, HR-HPV, or low-risk HPV (LR-HPV) infection status at the $t-1$ visit were included in the Cox models as time-varying exposures, with time discretized into 6-month intervals $[18,19]$. If one HPV result was missing, the previous visit result was carried 
forward, but if more than one HPV result was missing, they remained missing. Because HIV seroconversion was an uncommon event, estimated coefficients from the fitted Cox models were interpreted as estimating log risk ratios.

To examine the association between HPV natural history and HIV acquisition, Cox models were fit using the following four-level covariate: HPV negative at $t-2$ and $t-1$ (two visits before the HIV seroconversion visit); HPV persistence only, defined as type-specific HPV positivity at both $t-2$ and $t-1$ specific ; HPV clearance only, defined as type-HPV positivity at $t-2$ followed by loss of detection of that type at $t-1$; and both HPV persistence and clearance, defined as concurrent cleared and persistent infections since men can have more than one genotype infection. Because definitions vary in the literature, alternate comparisons for clearance and persistence were also analyzed:men with cleared infections at $t-1$ regardless of persistence or acquisition of other HPV infections compared to HPVnegative and men with persistent infections at $t-2$ regardless of clearance or acquisition of other HPV infections compared to HPV-negative.

To assess whether circumcision modified associations between HPV infection and HIV acquisition, an interaction term was added to the Cox models and statistical tests of homogeneity (a priori alpha $=0.10$ ) were conducted. Cox models were also run separately for circumcised and uncircumcised men to examine whether point estimates differed qualitatively (Online Supplement Table 1, http://links.lww.com/QAD/A424). Variables associated with both HPV infection and HIV serocon-version in previous analyses $[8,10,12,20]$ were included as potential confounders in the final multivariate model, including time-varying HSV-2 serostatus and recent sexual behavior (sex in the past 6 months, but not always with a condom vs. no sex or sex, but always with a condom in the past 6 months), and baseline age, education, and employment status. It was decided a priori that time-varying circumcision status should be included in the multivariate model if it was determined not to be an effect measure modifier.

\section{Results}

\section{Population characteristics and risk of HIV acquisition}

Of the 2519 men included in this analysis, 61 acquired HIV by 30 months of follow-up $(2.4 \%)$. The median time between visits was 6 months [interquartile range (IQR) 6-8] and median length of follow-up was 30 months (IQR 30-30). Median age at enrollment was 20 years (IQR 19-22) and age was not associated with the risk of HIV acquisition (Table 1). Men randomized to the immediate circumcision arm were less likely to acquire HIV than those who remained uncircumcised until the end of the follow-up period (aHR $0.31 ; 95 \% \mathrm{CI}$ $0.16-0.62$ ). Men who reported not always using condoms in the past 6 months (aHR 2.03; 95\% CI 1.03-3.99) and those who were HSV-2-seropositive (aHR 1.98; 95\% CI 1.04-3.76) were more likely to seroconvert than those who always used condoms or who were HSV-2seronegative. 


\section{Human papillomavirus and subsequent risk of HIV acquisition}

Of the 61 men who seroconverted over follow-up, 36 were HPV-infected (61\%) and 27 were HR-HPV-infected (46\%) (Table 2). The risk of HIV acquisition was over twice as high among men with any-HPV [unadjusted HR (uHR) 2.55; 95\% CI 1.51-4.32] and HR-HPV (uHR 2.74; 95\% CI 1.66-4.79) at the previous visit compared to men who were HPVnegative. The highest risk of HIV acquisition was among men with HR-HPV, then LR-HPV, and lowest among uninfected men $(P=0.0003$ test for trend). This pattern was consistent among both circumcised and uncircumcised men (Fig. 1), and there was little evidence of effect measure modification by circumcision status (Online Supplement Table 1, http:// links.lww.com/QAD/A424; uHR 1.99; 95\% CI 1.06-3.77 among uncircumcised and uHR 2.68; 95\% CI 1.03-7.01 among circumcised men). After adjusting for circumcision and other potential confounding variables, the risk of HIV associated with any-HPV (aHR 1.72; 95\% CI 0.94-3.15) and HR-HPV infection (aHR 1.92; 95\% CI 0.96-3.87) was attenuated slightly. However, the risk of HIV acquisition increased significantly with each additional HPV genotype infection (aHR 1.27; 95\% CI 1.09-1.48). Men with three or more HPV infections had over three times the risk of HIV acquisition compared to men who were HPV-uninfected at the $t-1$ visit (aHR 3.45; 95\% CI 1.49-7.96).

Type-specific analyses focused on infection with vaccine-preventable HPV types 16 and 18 demonstrated that among the $36 \mathrm{HPV}$-infected men who HIV-seroconverted, only three were infected with both HPV-16 and 18, or either of them, alone at $t-1$ (Table 3). In contrast, most HIV seroconverters had both HPV-16 and 18, or either of them, concurrently with other HPV genotypes (33\%), or had only non-16/18 HPV genotypes (58\%). Compared to HPV-uninfected men, the relative risk of HIV acquisition increased in a stepwise fashion among men HPV-positive for only non-16/18 types, positive for only HPV-16, 18, or both, and positive for $16 / 18$ with other HPV genotypes.

\section{Human papillomavirus natural history and risk of HIV acquisition}

An increased risk of HIV acquisition was observed among men who had recently cleared all HPV infections (aHR 2.94; 95\% CI 1.13-7.57), had only persistent HPV infections (aHR 7.45; 95\% CI 2.20-25.37), or who had both cleared and persistent infections (aHR 3.72; 95\% CI 1.30-10.57), as compared to men who were HPV-uninfected at $t-1$ and $t-2$ (Table 4). Alternate natural history definitions (e.g. men with cleared infections regardless of HPV persistence or acquisition, and men with persistent infections regardless of HPV clearance or acquisition) resulted in a similar three times increased risk of HIV acquisition for both HPV clearance and persistence compared to HPV-uninfected men (Table 4; Online Supplement Fig. 1, http://links.lww.com/QAD/A424).

\section{Discussion}

Because recent evidence in women has emerged to suggest that specifically cleared HPV infections are strongly associated with risk of HIV acquisition and because data in men are extremely limited, we have now analyzed HPV data collected at 6-month intervals for 2 years in the Kisumu male circumcision trial to determine if HPV infection and natural history are independently associated with risk of HIV. Incidence of HIV was highest among 
men with persistent and/or recently cleared HPV infections; these men had at least three times the risk of HIV compared to HPV-uninfected men, independent of HSV-2 and other potential confounding factors. We found no clear evidence that circumcision modifies the association between penile HPV infection and risk of HIV acquisition, although circumcision was strongly associated with acquisition of HIV. Each increase in the number of concurrent HPV infections was associated with a 27\% increased risk of HIV acquisition. Our results indicate that HPV infection is an important risk factor for HIV acquisition in men that needs to be explored further and accounted for in HIV-prevention studies.

Our analysis of longitudinal data from an RCT is consistent with previous studies in men and women showing an increased risk of HIV infection among those with anogenital HPV infection [3-7,9]. In the few studies that have examined the natural history of HPV infection, the highest HIV risk was observed among individuals with loss of HPV detection (i.e. clearance) compared to those with no HPV or persistent HPV infections [3,6,7]. Our estimate for risk of HIV associated with penile HPV clearance falls within the range of published relative risk estimates [1.67 (1.03-2.70) to 5.4 (2.9-9.9)] [3,6,7]. Unlike previous studies, however, we also found a similarly high risk of HIV among men with persistent HPV infections. A recent case-control analysis of penile HPV infection in predominantly married men from Rakai, Uganda, found that the relative risk of HIV associated with anytype persistent HPV was similar to the relative risk of HIV associated with HPV clearance, although both estimates were nonsignificant [7]. Similar to the present study, slightly different categorizations of HPV natural history resulted in somewhat different effect estimates.

Despite differences across studies, the consistently observed association of an increased risk of HIV acquisition with HPV clearance indicates that the immune-regulatory mechanism associated with the clearance of HPV may also facilitate HIV acquisition. In a study of foreskin samples, men who cleared HPV had significantly higher density of dendritic cells, which deliver HIV virions to $\mathrm{CD} 4^{+} \mathrm{T}$ cells, a potential explanation for how HPV clearance might increase susceptibility to HIV seroconversion [7]. However, other plausible mechanisms have been hypothesized, as reviewed in two recent meta-analyses of risk of HIV associated with HPV infection [21,22]. Moreover, the risk of HIV acquisition associated with HPV is unlikely to be fully attributable to confounding by sexual behaviour, given that effect estimates were similar across strata of recent sexual partners [risk per HPV infection among men with $\leq$ recent partner: aHR 1.36 (1.15-1.60) vs. + 2 recent partners: aHR 1. 25 (0.91-1.73)].

It is important to note limitations of our study and ways in which it differs from previous publications. First, use of GP5+/6+ PCR primers for HPV detection in our study could result in misclassification of HPV clearance or persistence because they may detect fewer multiple infections than other L1 primers $[23,24]$. Second, use of a 6-month interval could potentially underestimate the true risk of HIV if the association with HPV infection or clearance events is time-dependent and our measurement was outside the most biologically relevant window. However, given the 3-month period of uncertainty between the true date of HIV acquisition and serology-based detection, our use of 6-month time-lagged models ensures that HPV exposure occurred before HIV acquisition. In addition, our results are similar to our baseline 
HPV analysis and to a previous study using up to 1-year testing intervals [7,8]. Finally, we defined HPV natural history based on the two time-points prior to HIV seroconversion, potentially missing earlier clearance or persistence events and not distinguishing between short and long-term HPV persistence. However, we chose to model HPV events that were closest in time to HIV acquisition and use time-varying adjustment to account for HPV status at earlier study visits.

Uncircumcised men tend to have more STI co-infections, higher numbers of HIV target cells, and a different penile immune environment as compared to circumcised men [25-27]. Therefore, we examined whether the risk of HIV associated with HPV infection could differ by circumcision status. Our HIV risk estimates associated with HPV differed only slightly and the $P$-values for interaction terms (any-HPV* cir-circumcision $P=0.92$, and HR-HPV circumcision $P=0.18$ ) were nonsignificant. Despite the possibility of limited power to reliably detect effect measure modification due to the modest number of HIV cases, we conclude that estimates of HIV risk were not qualitatively or quantitatively different between circumcised and uncircumcised men.

There are currently two vaccines that provide near $100 \%$ protection against persistent infection with HPV-16 and 18, two highly prevalent genotypes that are responsible for $70 \%$ of invasive cervical cancer cases and a relatively smaller fraction of other HPV-associated cancers. This raises the question as to whether individuals who receive HPV vaccination will have the added benefit of lowering risk of HIV infection [5,21]. Since there are currently no data to directly estimate this, we used our observational data to examine risk of HIV acquisition among men infected with vaccine-preventable types 16/18, taking into account other concurrent HPV infections. Men with only HPV-16/18 were twice as likely to acquire HIV infection compared to men who were HPV-negative, but these men represent a minority of our population. Most HIV seroconverters had only non-16/18 genotype infections or had HPV-16/18 in addition to other HPV types, and thus would still have HPV even if vaccinated against HPV-16/18. However, we did find that HIV acquisition was twice as high among men with HPV-16/18 in addition to other HPV types as compared to men with only non-16 and 18 infections. The effect of cross-protection against other HR-HPV types in existing vaccines or a future nonavalent vaccine was not investigated here. In addition, further research is needed to understand the influence of HPV genotype independent of number of HPV infections.

Our findings that recently cleared and persistent HPV infections are associated with a modest increased risk of HIV acquisition, regardless of circumcision status, HSV-2 infection, and other potential confounding factors, are generalizable to other populations of young, predominately nonmarried, sexually active men from sub-Saharan Africa. In populations that are at high risk for HIV infection, HPV infection is common, with prevalence estimates over 50\% [12]. Taken together, these findings highlight the need for further research on potential immunological or behavioral mechanisms underlying these observational findings. Furthermore, although our data and others may suggest a potential reduction in HIV risk with a decrease in HPV infection via prophylactic HPV vaccination, we must be cautious in interpreting these findings in a population with a high prevalence of known HIV risk factors that would not be affected by HPV vaccination, such as HSV-2 
seropositivity, multiple genital infections, and other sexual risk behaviors such as condom use [28,29].

\section{Supplementary Material}

Refer to Web version on PubMed Central for supplementary material.

\section{Acknowledgments}

We would like to thank the young men of Kisumu who volunteered to participate in this study and all of the UNIM staff, especially the late Dr Jeckoniah O. Ndinya-Achola. Also, we thank Chelu Mfalila, Norma Pugh, and Dr Corette Parker for their assistance with the database.

All authors have contributed to, reviewed and approved the final manuscript. Author contributions: study conception and design (A.F.R., M.G.H., K.A., R.C.B., J.S.S., S.M.); study conduct, data collection, or laboratory analysis (K.A., E.N., R.C.B., C.J.L.M.M., P.J.F.S., S.M.); data analysis and interpretation (A.F.R., M.G.H., L.M., J.S.S.); manuscript preparation (A.F.R.); critical review of manuscript (A.F.R., M.G.H., L.M., K.A., D.M.B., E.N., R.C.B., C.J.L.M.M., P.J.F.S., J.S.S., S.M.).

Funding sources: This research was supported by the National Cancer Institute, National Institutes of Health (grant R01 CA1 14773-04) and the UNC Center for AIDS Research (grant P30 AI050410 from the National Institute of Allergy and Infectious Diseases, National Institutes of Health). The randomized controlled trial was supported by the Division of AIDS, National Institute of Allergy and Infectious Diseases, National Institutes of Health (grant AI50440), the Canadian Institutes of Health Research, and the Chicago Developmental Center for AIDS Research (D-CFAR), an NIH funded program (P30 AI 082151), which is supported by the following NIH Institutes and Centers (NIAID, NCI, NIMH, NIDA, NICHD, NHLBI, NCCAM). A.F.R. was supported by an NIH postdoctoral training grant in cancer epidemiology (grant T32 CA009314).

\section{References}

1. Bosch FX, Lorincz A, Munoz N, Meijer CJ, Shah KV. The causal relation between human papillomavirus and cervical cancer. J Clin Pathol. 2002; 55:244-265. [PubMed: 11919208]

2. Walboomers JM, Jacobs MV, Manos MM, Bosch FX, Kummer JA, Shah KV, et al. Human papillomavirus is a necessary cause of invasive cervical cancer worldwide. J Pathol. 1999; 189:1219. [PubMed: 10451482]

3. Averbach SH, Gravitt PE, Nowak RG, Celentano DD, Dunbar MS, Morrison CS, et al. The association between cervical human papillomavirus infection and HIV acquisition among women in Zimbabwe. AIDS. 2010; 24:1035-1042. [PubMed: 20397287]

4. Auvert B, Lissouba P, Cutler E, Zarca K, Puren A, Taljaard D. Association of oncogenic and nononcogenic human papillomavirus with HIV incidence. J Acquir Immune Defic Syndr. 2010; 53:111-116. [PubMed: 19779357]

5. Auvert B, Marais D, Lissouba P, Zarca K, Ramjee G, Williamson AL. High-risk human papillomavirus is associated with HIV acquisition among South African female sex workers. Infect Dis Obstet Gynecol. 2011; 2011:692012. [PubMed: 21804752]

6. Smith-McCune KK, Shiboski S, Chirenje MZ, Magure T, Tuveson J, Ma Y, et al. Type-specific cervico-vaginal human papillomavirus infection increases risk of HIV acquisition independent of other sexually transmitted infections. PLoS One. 2014; 5:e10094. [PubMed: 20386706]

7. Tobian AA, Grabowski MK, Kigozi G, Redd AD, Eaton KP, Serwadda D, et al. Human papillomavirus clearance in men is associated with HIV acquisition and increased foreskin dendritic cell density in Rakai, Uganda. J Infect Dis. 2013; 207(11):1713-1722. [PubMed: 23345339]

8. Smith JS, Moses S, Hudgens MG, Parker CB, Agot K, Maclean I, et al. Increased risk of HIV acquisition among Kenyan men with human papillomavirus infection. J Infect Dis. 2010; 201:16771685. [PubMed: 20415595]

9. Chin-Hong PV, Husnik M, Cranston RD, Colfax G, Buchbinder S, Da Costa M, et al. Anal human papillomavirus infection is associated with HIV acquisition in men who have sex with men. AIDS. 2009; 23:1135-1142. [PubMed: 19390418] 
10. Bailey RC, Moses S, Parker CB, Agot K, Maclean I, Krieger JN, et al. Male circumcision for HIV prevention in young men in Kisumu, Kenya: a randomised controlled trial. Lancet. 2007; 369:643656. [PubMed: 17321310]

11. Gray RH, Serwadda D, Kong X, Makumbi F, Kigozi G, Gravitt PE, et al. Male circumcision decreases acquisition and increases clearance of high-risk human papillomavirus in HIV-negative men: a randomized trial in Rakai, Uganda. J Infect Dis. 2010; 201:1455-1462. [PubMed: 20370483]

12. Smith JS, Backes DM, Hudgens MG, Bailey RC, Veronesi G, Bogaarts M, et al. Prevalence and risk factors of human papillomavirus infection by penile site in uncircumcised Kenyan men. Int J Cancer. 2010; 126:572-577. [PubMed: 19626601]

13. Smith JS, Moses S, Hudgens MG, Agot K, Franceschi S, Maclean IW, et al. Human papillomavirus detection by penile site in young men from Kenya. Sex Transm Dis. 2007; 34:928934. [PubMed: 17621251]

14. van den Brule AJ, Pol R, Fransen-Daalmeijer N, Schouls LM, Meijer CJ, Snijders PJ. GP5+/6+ PCR followed by reverse line blot analysis enables rapid and high-throughput identification of human papillomavirus genotypes. J Clin Microbiol. 2002; 40:779-787. [PubMed: 11880393]

15. Snijders, PJF.; Van den Brule, AJC.; Jacobs, MV.; Pol, RP.; Meijer, CJLM. HPV DNA detection and typing in cervical scrapes by general primer GP5+/6+ PCR.. In: Davy, CE.; Doorbar, J., editors. Methods in molecular medicine: human papillomaviruses: methods and protocols Totowa. Humana Press; 2005. p. 101-114.

16. Snappin SM, Jiang Q, Iglewicz B. Illustrating the impact of a time-varying covariatewith an extended Kaplan-Meier estimator. Am Stat. 2005; 59:301-308.

17. Allison, P. Survival analysis using SAS: a practical guide. 2nd ed.. SAS Institute; Cary, NC: 2010.

18. Breslow N. Covariance analysis of censored survival data. Biometrics. 1974; 30:89-99. [PubMed: 4813387]

19. Hertz-Picciotto I, Rockhill B. Validity and efficiency of approximation methods for tied survival times in Cox regression. Biometrics. 1997; 53:1151-1156. [PubMed: 9333345]

20. Rositch AF, Hudgens MG, Backes DM, Moses S, Agot K, Nyagaya E, et al. Vaccine-relevant human papillomavirus (HPV) infections and future acquisition of high-risk HPV types in men. $\mathbf{J}$ Infect Dis. 2012; 206:669-677. [PubMed: 22711906]

21. Houlihan CF, Larke NL, Watson-Jones D, Smith-McCune KK, Shiboski S, Gravitt PE, et al. Human papillomavirus infection and increased risk of HIV acquisition. A systematic review and meta-analysis. AIDS. 2012; 26:2211-2222. [PubMed: 22874522]

22. Lissouba P, Van de Perre P, Auvert B. Association of genital human papillomavirus infection with HIV acquisition: a systematic review and meta-analysis. Sex Transm Infect. 2013; 89(5):350-356. [PubMed: 23761216]

23. Camargo M, Soto-De Leon S, Sanchez R, Munoz M, Vega E, Beltran M, et al. Detection by PCR of human papillomavirus in Colombia: comparison of GP5+/6+ and MY09/11 primer sets. J Virol Methods. 2011; 178:68-74. [PubMed: 21884728]

24. Gravitt PE, Peyton CL, Alessi TQ, Wheeler CM, Coutlee F, Hildesheim A, et al. Improved amplification of genital human papillomaviruses. J Clin Microbiol. 2000; 38:357-361. [PubMed: 10618116]

25. Morris BJ, Wamai RG. Biological basis for the protective effect conferred by male circumcision against HIV infection. Int J STD AIDS. 2012; 23:153-159. [PubMed: 22581866]

26. Bailey RC, Plummer FA, Moses S. Male circumcision and HIV prevention: current knowledge and future research directions. Lancet Infect Dis. 2001; 1:223-231. [PubMed: 11871509]

27. Tobian AA, Serwadda D, Quinn TC, Kigozi G, Gravitt PE, Laeyendecker O, et al. Male circumcision for the prevention of HSV-2 and HPV infections and syphilis. N Engl J Med. 2009; 360:1298-1309. [PubMed: 19321868]

28. Chen L, Jha P, Stirling B, Sgaier SK, Daid T, Kaul R, et al. Sexual risk factors for HIV infection in early and advanced HIV epidemics in sub-Saharan Africa: systematic overview of 68 epidemiological studies. PLoS One. 2007; 2:e1001. [PubMed: 17912340] 
29. Rositch AF, Gravitt PE, Smith JS. Growing evidence that HPV infection is associated with an increase in HIV acquisition:exploring the issue of HPV vaccination. Sex Transm Infect. 2013; 89:357. [PubMed: 23858496] 

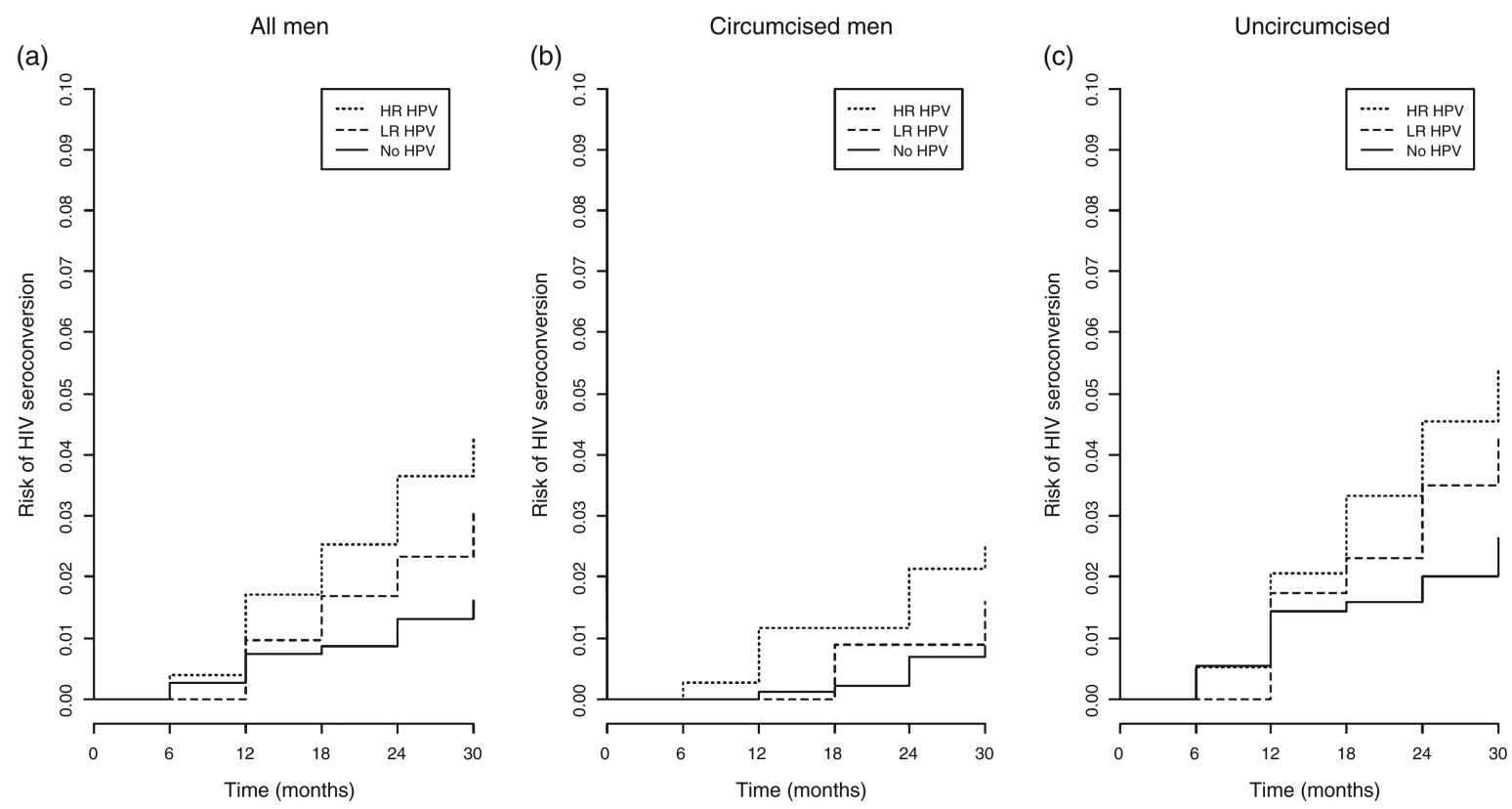

Fig. 1. Kaplan-Meier curves displaying the cumulative risk of HIV seroconversion among men with no HPV infection, LR-HPV types only, and HR-HPV infection at $t-1$ among (a) total population; (b) circumcised men; and (c) uncircumcised men HPV, human papillomavirus; HR-HPV, high-risk human papillomavirus; LR-HPV, low-risk human papillomavirus. 


\section{Table 1}

Participant characteristics at their first study visit stratified by HIV serostatus at end of study follow-up.

\begin{tabular}{|c|c|c|c|c|}
\hline & HIV-negative $(n=2458)$ & HIV-seroconverters $(n=61)$ & uHR $(95 \%$ CI $)$ & aHR $(95 \%$ CI) \\
\hline \multicolumn{5}{|c|}{$N(\%)$ or median (IQR) } \\
\hline Age in years at baseline & $20(19-22)$ & $20(19-21)$ & $0.98(0.83-1.15)$ & $0.94(0.79-1.13)$ \\
\hline \multicolumn{5}{|l|}{ Randomization group } \\
\hline Uncircumcised & $1218(50 \%)$ & $47(77 \%)$ & 1 & 1 \\
\hline Circumcised & $1240(50 \%)$ & $14(23 \%)$ & $0.35(0.20-0.61)$ & $0.31(0.16-0.62)$ \\
\hline \multicolumn{5}{|l|}{ Employment status } \\
\hline Unemployed & $1580(64 \%)$ & $31(51 \%)$ & 1 & 1 \\
\hline Employed & $878(36 \%)$ & $30(49 \%)$ & $2.05(1.16-3.61)$ & $1.72(0.91-3.25)$ \\
\hline \multicolumn{5}{|l|}{ Education } \\
\hline Any secondary or above & $1610(66 \%)$ & $33(54 \%)$ & 1 & 1 \\
\hline Less than secondary & $848(34 \%)$ & $28(46 \%)$ & $1.97(1.12-3.48)$ & $1.27(0.68-2.37)$ \\
\hline \multicolumn{5}{|l|}{ Sexual behavior, previous 6 months } \\
\hline No sex or sex always with condoms ${ }^{a}$ & $843(34 \%)$ & $14(23 \%)$ & 1 & 1 \\
\hline Sex not always with condoms & $1613(66 \%)$ & $47(77 \%)$ & $2.55(1.32-4.92)$ & $2.03(1.03-3.99)$ \\
\hline \multicolumn{5}{|l|}{ Any-HPV DNA } \\
\hline No & $1279(52 \%)$ & $21(34 \%)$ & 1 & 1 \\
\hline Yes & $1179(48 \%)$ & $40(66 \%)$ & $2.55(1.51-4.32)$ & $1.72(0.94-3.15)$ \\
\hline \multicolumn{5}{|l|}{ Herpes simplex virus 2} \\
\hline Seronegative & $1547(74 \%)$ & $32(62 \%)$ & 1 & 1 \\
\hline Seropositive & $549(26 \%)$ & $19(37 \%)$ & $2.04(1.17-3.54)$ & $1.98(1.04-3.76)$ \\
\hline \multicolumn{5}{|l|}{ Gonorrhea or Chlamydia } \\
\hline No & $2308(94 \%)$ & $57(93 \%)$ & 1 & 1 \\
\hline Yes & $147(6 \%)$ & $4(7 \%)$ & $0.95(0.23-3.92)$ & $0.99(0.24-4.18)$ \\
\hline
\end{tabular}

aHR, adjusted hazard ratio; CI, confidence interval; HPV, human papillomavirus; IQR, interquartile range; uHR, unadjusted hazard ratio. Missing data: employment $(n=0)$, education $(n=0)$, recent sexual behavior $(n=2)$, herpes simplex virus $2(n=372)$, gonorrhea and chlamydia $(n=3)$.

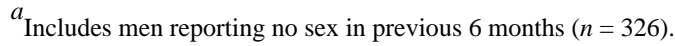


Table 2

HPV infection status at the visit prior to HIV seroconversion.

\begin{tabular}{lrccc}
\hline & No. of HIV seroconverters & Incidence rate (95\% CI) & uHR (95\% CI) & aHR (95\% CI) \\
\hline HPV infection & & & & \\
No HPV & 23 & $0.54(0.32-0.76)$ & 1 & 1 \\
$\quad$ Any-HPV & 36 & $1.33(0.90-1.76)$ & $2.55(1.51-4.32)$ & $1.72(0.94-3.15)$ \\
No HPV & 23 & $0.54(0.32-0.76)$ & 1 & 1 \\
$\quad$ LR-HPV & 9 & $1.10(0.34-1.85)$ & $2.04(0.95-4.40)$ & $1.25(0.45-3.42)$ \\
$\quad$ HR-HPV & 27 & $1.54(0.94-2.14)$ & $2.74(1.66-4.79)$ & $1.92(0.96-3.87)$ \\
Number of HPV infections & & & & 1 \\
0 types & 23 & $0.54(0.32-0.76)$ & & 1 \\
$\quad$ 1-2 types & 22 & $1.01(0.55-1.48)$ & $1.70(0.90-3.23)$ & $1.41(0.64-3.13)$ \\
$\quad 3$ types & 14 & $2.68(1.38-3.99)$ & $4.92(2.55-9.47)$ & $3.45(1.49-7.96)$ \\
Risk per additional genotype & 59 & & $1.33(1.22-1.48)$ & $1.27(1.09-1.48)$ \\
\hline
\end{tabular}

aHR, adjusted hazard ratio; CI, confidence interval; HR-HPV, high-risk human papillomavirus; LR-HPV, low-risk human papillomavirus; uHR, unadjusted hazard ratio.

${ }^{a}$ Incidence rates are expressed as infections per 1000 person-months. 
Table 3

Infection with vaccine and nonvaccine-preventable HPV types and risk of HIV seroconversion.

\begin{tabular}{lrcc}
\hline & No. of HIV seroconverters & uHR (95\% CI) & aHR (95\% CI) \\
\hline HPV-negative & 23 & 1 & 1 \\
HPV-positive with non-16/18 & 21 & $2.38(1.23-4.34)$ & $1.38(0.69-2.78)$ \\
HPV-16 and/or 18 positive only & 3 & $2.07(0.45-8.87)$ & $2.11(0.51-9.05)$ \\
HPV-16 and/or 18 with other types & 12 & $5.69(2.74-11.93)$ & $4.28(1.95-9.33)$ \\
\hline
\end{tabular}

aHR, adjusted hazard ratio; CI, confidence interval; HPV, human papillomavirus; uHR, unadjusted hazard ratio. 
Table 4

HPV natural history and subsequent risk of HIV seroconversion.

\begin{tabular}{lcccc}
\hline & $\begin{array}{c}\text { No. of HIV } \\
\text { seroconverters }\end{array}$ & $\begin{array}{c}\text { Incidence rate per 1000 } \\
\text { person-months (95\% CI) }\end{array}$ & uHR (95\% CI) & aHR (95\% CI) \\
\hline $\begin{array}{l}\text { HPV natural history } \\
\text { HPV-uninfected }\end{array}$ & 20 & $0.44(0.27-0.60)$ & 1 & 1 \\
$\quad$ Clearance, only & 19 & $0.85(0.47-1.23)$ & $2.12(1.05-4.33)$ & $2.94(1.13-7.57)$ \\
$\quad$ Persistence, only & 4 & $2.50(0.07-4.93)$ & $5.17(1.68-15.84)$ & $7.45(2.20-25.37)$ \\
Clearance + Persistence & 11 & $2.64(1.09-4.18)$ & $5.76(2.57-12.87)$ & $3.72(1.30-10.57)$ \\
Alternate natural history definitions & & & & \\
HPV-uninfected & 20 & $0.44(0.27-0.60)$ & 1 & 1 \\
Cleared infections & 30 & $1.18(0.75-1.60)$ & $2.78(1.43-5.39)$ & $3.05(1.34-6.97)$ \\
HPV-uninfected & 20 & $0.44(0.27-0.60)$ & 1 & 1 \\
Persistent infections & 15 & $1.92(1.06-2.77)$ & $4.41(2.28-8.47)$ & $3.27(1.59-6.72)$ \\
\hline
\end{tabular}

aHR, adjusted hazard ratio; CI, confidence interval; HPV, human papillomavirus; uHR, unadjusted hazard ratio.

${ }^{a}$ Mutually exclusive categories were defined as HPV-negative at $t-2$ and $t-1 ; \mathrm{HPV}$ persistence only, defined as type-specific HPV positivity at both $t-2$ and $t-1$; HPV clearance only, defined as type-specific HPV positivity at $t-2$ followed by loss of detection of that type at $t-1$; and HPV persistence and clearance, defined as concurrent cleared and persistent infections since men can have more than one type infection with different natural histories at $t-2$ and $t-1$. 